\title{
Observation of topological bound states in a double Su-Schrieffer-Heeger chain composed of split ring resonators
}

\author{
Zhiwei Guo $\odot,{ }^{1, *}$ Jun Jiang, ${ }^{2}$ Haitao Jiang,,${ }^{1 \dagger}$ Jie Ren $\odot,{ }^{3}$ and Hong Chen ${ }^{1, *}$ \\ ${ }^{1}$ MOE Key Laboratory of Advanced Micro-Structured Materials, School of Physics Science and Engineering, \\ Tongji University, Shanghai 200092, China \\ ${ }^{2}$ School of Automotive Studies, Tongji University, Shanghai 210804, China \\ ${ }^{3}$ Center for Phononics and Thermal Energy Science, China-EU Joint Center for Nanophononics, \\ Shanghai Key Laboratory of Special Artificial Microstructure Materials and Technology, \\ School of Physics Science and Engineering, Tongji University, Shanghai 200092, China
}

(Received 2 September 2020; revised 20 January 2021; accepted 25 January 2021; published 8 February 2021)

\begin{abstract}
The observation of abundant topological properties in optical platforms has attracted people's attention due to their great advantages in fundamental topological research and practical applications. Here, we use a double $\mathrm{Su}-\mathrm{Schrieffer-Heeger} \mathrm{(SSH)} \mathrm{chain} \mathrm{structure} \mathrm{composed} \mathrm{of} \mathrm{split} \mathrm{ring} \mathrm{resonators} \mathrm{(SRRs)} \mathrm{to} \mathrm{mimic} \mathrm{a} \mathrm{Kitaev} \mathrm{model.}$ By properly tuning the orientation angles of the slits of the SRRs, we can ingeniously design the complex coupling distribution. We experimentally measure the topological phase transition of the double SSH model by adjusting the distance between the two chains and observe the topological bound states in the topological nontrivial phase. Importantly, inserting a trivial structure into the topological chain, we show the coupling of two topological bound states. By increasing the length of the inserted trivial chain, we observe that two topological bound states gradually approach each other and degenerate in the critical state. In addition, we theoretically study the controllable topological bound states and exceptional point physics formed by topological bound states. Our findings not only clearly show the establishing process of the photonic bound states in a double SSH chain, but also may facilitate some applications such as sensors, energy transfer, and switch with topological protection.
\end{abstract}

DOI: 10.1103/PhysRevResearch.3.013122

\section{INTRODUCTION}

Topological property adds a degree of freedom to manipulate electrons in condensed-matter systems [1,2]. Recently, this concept has been transferred to photonic systems to control the flow of photons [3,4]. With the development of metamaterials, optical systems provide unprecedented ability to study many topological phenomena that are difficult to observe in condensed-matter systems [5-13]. The extensive study of the topological phenomena in optics leads to the formation of topological photonics [3,4], which opens a path to manipulate light [14-20]. For example, the Su-SchriefferHeeger (SSH) model is one of the basic geometries to study topological properties of the organic molecule polyacetylene [21]. To date, many photonic analogs of topological structures based on this model are proposed to study the topological excitations of light [22-26] and the properties of photonic structures based on the SSH model have been extended to the

\footnotetext{
${ }^{*}$ Corresponding author: 2014guozhiwei@ tongji.edu.cn

†Corresponding author: jiang-haitao@tongji.edu.cn

‡Corresponding author: hongchen@ tongji.edu.cn
}

Published by the American Physical Society under the terms of the Creative Commons Attribution 4.0 International license. Further distribution of this work must maintain attribution to the author(s) and the published article's title, journal citation, and DOI.
non-Hermitian [27,28], nonlinear [20,28-30], and quasiperiodic systems [31-33].

Besides the SSH model, the Kitaev model, which is one of the basic geometries for describing topological superconductors, has recently attracted researchers' great attention, owing to the existence of unpaired Majorana fermions in the topological nontrivial phase [34-41]. Remarkably, the Majorana fermions may have some important applications in quantum computation and information $[42,43]$. However, it is very difficult to realize this topological superconductor in condensed-matter systems because the required experimental conditions are very critical [34-45]. In analogy to the condensed-matter systems, some optical structures are put forward to demonstrate the photonic Majorana states [45-54]. Nevertheless, even in optical systems the experimental realization of photonic Majorana states is still very challenging. The main obstacle comes from the complex coupling distributions in the Kitaev model [34]. In this paper, based on a double SSH chain composed of split ring resonators (SRRs), we delicately tune the coupling distributions in the Kitaev model and experimentally observe the topological phase transition and the photonic bound states. Here, the role of particle-hole excitation of the Kitaev model is taken by the two chain degrees of freedom. In addition, it should be noted that the bound state of the mimetic optical Kitaev chain is not a Majorana fermion, but a number conserved state. Therefore, the bound state in the photonic Kitaev chain is called the topological bound state, not the Majorana fermion. Nevertheless, the mimetic Kitaev 
chain provides a specific experimental implementation system, which can map the phase diagram of the Kitaev chain and the spatial structure of its localized bound states $[39,49]$. In addition, considering the quasiperiodic modulation of on-site frequency of resonators, we find that the confined position of topological bound state can be controlled. Specifically, based on the topological phase diagram of photonic Kitaev chain, we study the interaction of two topological bound states at the interface between the topological phase and the trivial phase. Similar to the level splitting of optical modes [55], we find that the interaction between two topological bound states can be effectively controlled by tuning the distance between them. By increasing the coupling distance, we observe that two topological bound states gradually approach each other. Considering the composite Kitaev chain satisfying parity-time (PT) symmetry, we also theoretically propose a sensor with high sensitivity and topological protection. Therefore, Kitaev chains based on SRRs not only provide a flexible platform for the study of topological transition and topological bound states, but also may be used to reveal more interesting topological properties of the coupled topological bound states.

\section{MODEL}

With the tight-binding formalism, the dynamics in the standard Kitaev configuration can be expressed by the following Hamiltonian [34]:

$$
\begin{aligned}
H= & \sum_{j}\left[-t\left(c_{j}^{\dagger} c_{j+1}+c_{j+1}^{\dagger} c_{j}\right)-\mu\left(c_{j}^{\dagger} c_{j}-\frac{1}{2}\right)\right. \\
& \left.+\left(\Delta c_{j}^{\dagger} c_{j+1}^{\dagger}+\Delta^{*} c_{j+1} c_{j}\right)\right],
\end{aligned}
$$

where $c_{j}^{\dagger}$ and $c_{j}$ are the creation and annihilation operators at site $j$, respectively, while $-t$ is a hopping amplitude. $\mu$ is a chemical potential and $\Delta$ is the order parameter under meanfield approximation. The creation and annihilation operators can be written as

$$
c_{j}=\frac{1}{2}\left(\gamma_{j 1}+i \gamma_{j 2}\right), c_{j}^{\dagger}=\frac{1}{2}\left(\gamma_{j 1}-i \gamma_{j 2}\right),
$$

respectively. After Eq. (2) is put into Eq. (1), we can get the Hamiltonian of Kitaev model under the Majorana expression

$H=\frac{i}{2}\left\{\sum_{j=1}^{N}\left(\kappa_{3} \gamma_{j A} \gamma_{j B}\right)+\sum_{j=1}^{N-1}\left[\kappa_{1} \gamma_{j B} \gamma_{(j+1) A}+\kappa_{2} \gamma_{j A} \gamma_{(j+1) B}\right]\right\}$,

where $A$ and $B$ represent the upper and lower chains of the Kitaev double chain, respectively. $N$ denotes the total number of sites in each single chain. For the photonic Kitaev model, $\kappa_{1}=t+\Delta$ or $\kappa_{2}=-t+\Delta$ denotes the diagonal coupling coefficient between the two chains and $\kappa_{3}=-\mu$ is the interchain vertical coupling coefficient, as is schematically shown in Fig. 1(a). In this standard Kitaev model, it is required that $\kappa_{1}$ and $\kappa_{2}$ are much larger than the intrachain coupling coefficient denoted by $\kappa_{4}$ [34]. However, in optical systems, the near-field coupling strength is often directly related to the distance between two adjacent resonators and thereby $\kappa_{1}$, $\kappa_{2}$, and $\kappa_{4}$ are always in the same order of magnitude [55].
To overcome this problem, Poddubny et al. used polarization in different directions to construct a zigzag chain that is composed of plasmonic nanoparticles to mimic a topological Kitaev model [49,50]. In addition, the SSH ladder system acting as an analog of the Kitaev chain has been proposed based on the extended SSH model with long-range hopping [54]. Unlike previous studies, here we construct an effective Kitaev chain using the unique coupling characteristics of SRRs. Based on the microwave platform, we systematically study the topological phase transition, topological bound states, and the coupling effect of topological bound states.

When the Kitaev model is mapped into a classical system, each site can be replaced by two coupled resonators and the couplings between fermions in a superconductor are mapped by the near-field interaction between resonators. As a result, the Hamiltonian is rewritten from a two by two matrix to a four by four matrix. In momentum space representation, the Hamiltonian of Kitaev model for Bloch modes has the form

$$
H(k)=\left(\begin{array}{cccc}
0 & 0 & \kappa_{3} & W(k) \\
0 & 0 & P^{*}(k) & \kappa_{3} \\
\kappa_{3} & P(k) & 0 & 0 \\
W^{*}(k) & \kappa_{3} & 0 & 0
\end{array}\right)
$$

where $W=\kappa_{1}+\kappa_{2} e^{-i k}, \quad P=\kappa_{2}+\kappa_{1} e^{-i k}, \quad$ and $\quad k \quad$ is Bloch wave vector. Under a linear transformation $\left[U^{-1} H(k) U=H^{\prime}(k)\right]$, we propose an effective Kitaev model to study topological bound states. The linearly transformed matrix has the form $\left.U=\begin{array}{rrrl}1 & 0 & 0 & 0 \\ 0 & 0 & 0 & 1 \\ 0 & 0 & 1 & 0 \\ 0 & 1 & 0 & 0\end{array}\right)$, and the transformed Hamiltonian is $[35,38]$

$$
H^{\prime}(k)=\left(\begin{array}{cccc}
0 & W(k) & \kappa_{3} & 0 \\
W^{*}(k) & 0 & 0 & \kappa_{3} \\
\kappa_{3} & 0 & 0 & P(k) \\
0 & \kappa_{3} & P^{*}(k) & 0
\end{array}\right) .
$$

The corresponding schematic of the effective Kitaev chain is shown in Fig. 1(b). Intuitively, the effective model in Fig. 1(b) can be realized from Fig. 1(a) by exchanging the positions of 2 and 4 in the unit cell. In accordance with the standard Kitaev model, $\kappa_{1}$ and $\kappa_{2}$ in Fig. 1(b) should be much larger than $\kappa_{4}$. When $k=0$, the eigenvalues of $H^{\prime}$ are $E_{1}=$ $-\kappa_{1}-\kappa_{2}-\kappa_{3}, E_{2}=-\kappa_{1}-\kappa_{2}+\kappa_{3}, E_{3}=\kappa_{1}+\kappa_{2}+\kappa_{3}$, and $E_{4}=\kappa_{1}+\kappa_{2}-\kappa_{3}$. The band gap will be closed in the case that $\left|\kappa_{3}\right|=\left|\kappa_{1}+\kappa_{2}\right|$. The topological invariant of the Kitaev chain takes the form of $[34,36]$

$$
v=\operatorname{sgn}(|\mu|-2|\Delta|)=\operatorname{sgn}\left(\left|\kappa_{3}\right|-\left|\kappa_{1}+\kappa_{2}\right|\right),
$$

where $v=1(v=-1)$ corresponds to topological trivial (nontrivial) phase. From Fig. 1(c), we can find that the topological nontrivial phase in the case that $\left|\kappa_{3}\right|<\left|\kappa_{1}+\kappa_{2}\right|$ and the topological trivial phase in the case that $\left|\kappa_{3}\right|>\left|\kappa_{1}+\kappa_{2}\right|$ are separated by the red lines $\left(\left|\kappa_{3}\right|=\left|\kappa_{1}+\kappa_{2}\right|\right)$. These red lines correspond to the phase transition points (PTPs) [34]. For example, if we choose $\kappa_{1}=-0.11$ and $\kappa_{2}=0.24$, the topological transition will take place when $\kappa_{3}$ changes from -0.18 to -0.08 and the PTP corresponds to $\left|\kappa_{3}\right|=\kappa_{1}+\kappa_{2}=$ 
(a)

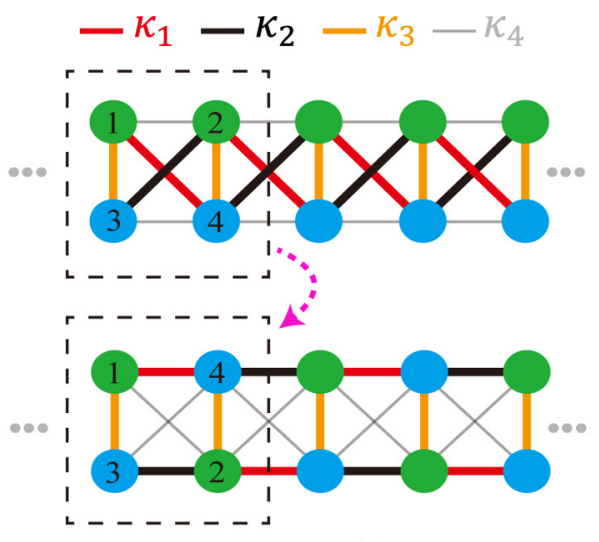

(d)

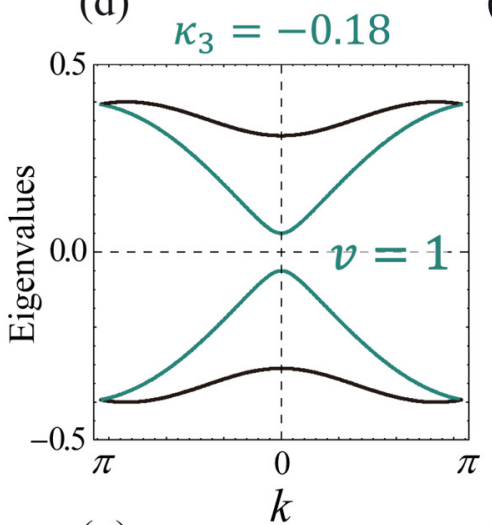

(g)

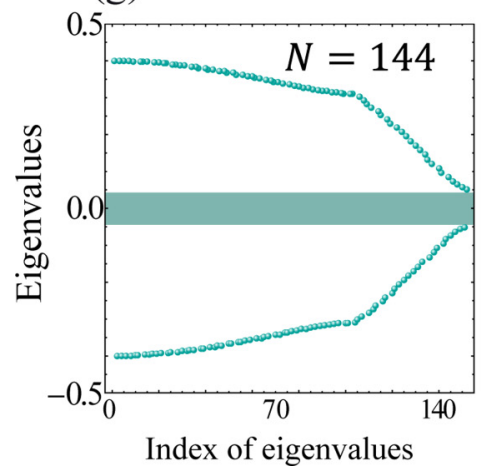

(e)

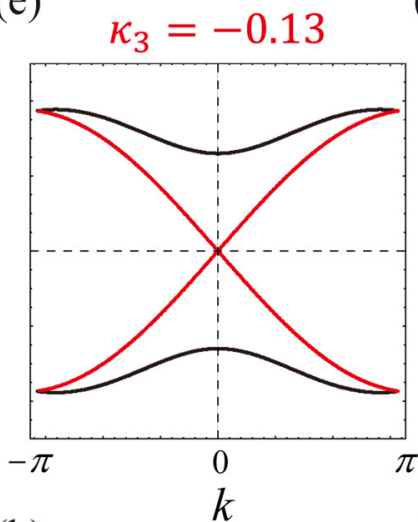

(h)

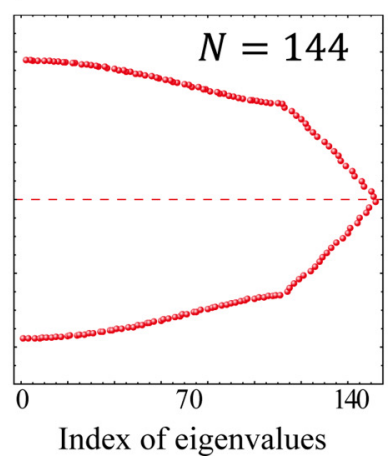

(c)

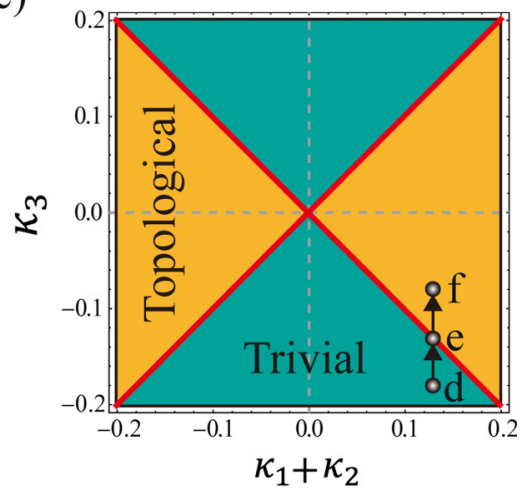

(f)

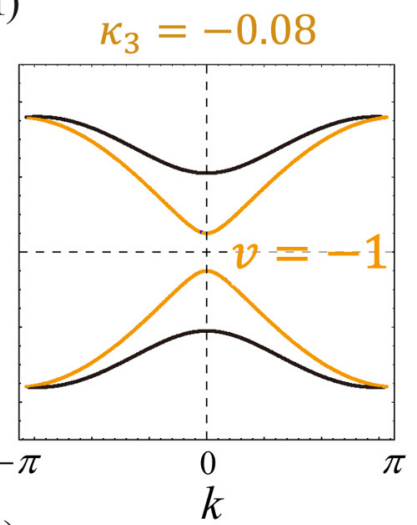

(i)

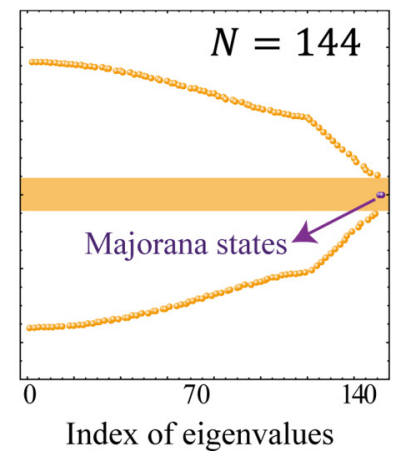

FIG. 1. (a) Schematic of the standard Kitaev model, in which $\kappa_{1}$ and $\kappa_{2}$ are much larger than $\kappa_{4}$. (b) Schematic of the effective Kitaev model that is derived by exchanging the positions of 2 and 4 in the unit cell of (a). (c) Phase diagram for the effective Kitaev chain. The topological trivial phase (green zone) and nontrivial phase (orange zone) are separated by the phase transition points (red lines). When $\kappa_{1}+\kappa_{2}=0.13$ and $\kappa_{4}=0.0025$, the band structures for $\kappa_{3}=-0.18$ (d) and $\kappa_{3}=-0.08$ (f) are topological trivial and nontrivial, respectively, while $\kappa_{3}=-0.13$ (e) corresponds to the transition case. (g)-(i) The eigenvalue spectra of the finite Kitaev chains with 144 resonators, which corresponds to the band diagrams in (d)-(f), respectively. The topological bound states in topological nontrivial structure are marked by the arrow in (i).

0.13 ; see $d \rightarrow e \rightarrow f$ in the fourth quadrant of Fig. 1(c). The corresponding band structures in three cases are shown in Figs. 1(d)-1(f), respectively. In the case $\kappa_{3}=-0.18$ the band gap corresponds to a topological trivial phase $(v=1)$. Nevertheless, in the case $\left|\kappa_{3}\right|=\kappa_{1}+\kappa_{2}=0.13$, the band gap is closed, which corresponds to a PTP. After $\kappa_{3}$ is increased to -0.08 , a band gap reopens and it corresponds to a topological nontrivial phase $(v=-1)$. Considering the finite structures with 144 resonators, the eigenvalues spectra in Figs. 1(g)-1(i) correspond to the band diagrams in Figs. 1(d)-1(f), respectively. For a topological nontrivial Kitaev chain corresponding to $\kappa_{3}=-0.08$, the eigenvalues spectrum is shown in Fig. 1(i). In this case we see that a pair of topological bound states exist in the band gap marked by the orange zone. However, for a finite topological trivial chain corresponding to $\kappa_{3}=-0.18$, there is no topological bound state in the band gap marked by the green zone, as is shown in Fig. 1(g).

\section{PHOTONIC KITAEV CHAIN AND PHASE TRANSITION}

Now we use two SRR chains to mimic the effective Kitaev chain in Fig. 1(b). The schematic of the two coupled SRRs with arbitrarily rotated angles in planar configuration is shown in Fig. 2(a). The thickness, height, inner radius, and the gap size of each SRR are $w=1.0 \mathrm{~mm}, h=5.0 \mathrm{~mm}$, $r=10 \mathrm{~mm}$, and $g=1.5 \mathrm{~mm}$, respectively (see more details in 


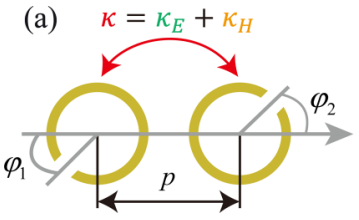

(b)

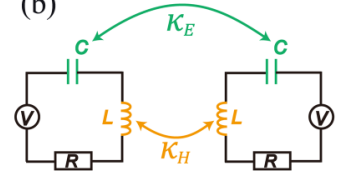

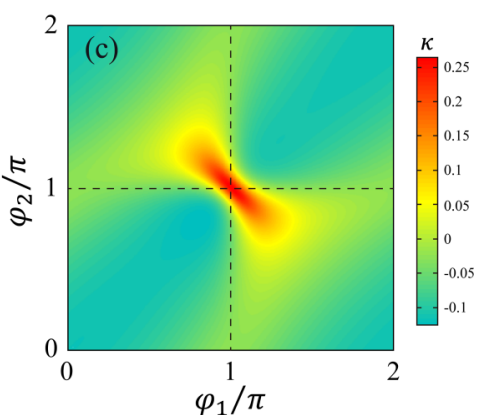

FIG. 2. (a) The coupling between two arbitrarily rotated SRRs is composed of magnetic and electric coupling. The rotating angles of the SRRs are $\varphi_{1}$ and $\varphi_{2}$ respectively. (b) Equivalent circuit for two coupling SRRs. (c). The dependence of total coupling coefficient $\kappa$ on the relevant angle between two SRRs when $p=24 \mathrm{~mm}$.

Appendix A). The resonance frequency of SRRs is $\omega_{0}=$ $1.836 \mathrm{GHz}$. In each SRR chain, the distance between two neighboring SRRs is denoted by $p$ and it is fixed to be 24 $\mathrm{mm}$. The total coupling coefficient $\kappa=\kappa_{E}+\kappa_{H}$ which is dominated either by its electric part $\kappa_{E}$ or its magnetic part $\kappa_{H}$ is directly related to the relevant angles between two SRRs (see more details in Appendix A). The coupling between two SRRs can be modeled by equivalent inductance-capacitanceresistance (LCR) circuit, which is shown in Fig. 2(b). The calculated relationship between rotating angles and the total coupling coefficient $\kappa$ is given in Fig. 2(c). The details of the dependence of the coupling coefficient on the relative rotation angle and the separating distance are given in Appendix A. Obviously, the coupling strength can be near zero at the proper rotation angles, although the distance between two rings is very short. So, the SRRs with arbitrarily rotated angles are very suitable for constructing the Kitaev model with complex coupling distribution in Fig. 1(b).

Figures 3(a) and 3(b) are the photo of the structure and the corresponding model, respectively. Compared to the disks or spheres, the coupling coefficients between SRRs are not only dependent on the separating distance, but also related to the relative rotation angle of the slits of two neighboring resonators [25]. So, the special coupling distributions of the Kitaev model can be realized based on SRRs. Moreover, because the distance between the two chains in the effective Kitaev model can be flexibly tuned, we can study the whole phase diagram from which we can identify the topological trivial and nontrivial phases and observe the establishment of topological bound states. In Fig. 3(a), the distance between the upper and lower SRR chains is represented by $d$. From Fig. 3(b), we can find that there are four kinds of coupling coefficients in the unit cell (marked by the green dashed rectangle) of the Kitaev model (see more details in Appendix A). In each SRR chain, when the relative rotation angle of the slits of two neighboring SRRs is $180^{\circ}$, the coupling between two SRRs is weak and $\kappa_{1}=-0.11$. However, when the relative rotation angle of the slits of two adjacent SRRs is $0^{\circ}$, the coupling between two SRRs is strong and $\kappa_{2}=0.24$. In addition, the vertical and diagonal coupling coefficients between the two SRR chains are represented by $\kappa_{3}$ and $\kappa_{4}$, respectively, which can be tuned by changing the distance $d$. It is interesting (a)

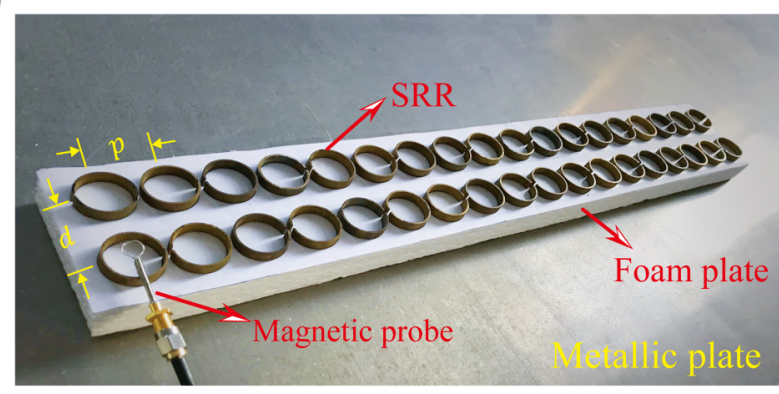

(b)

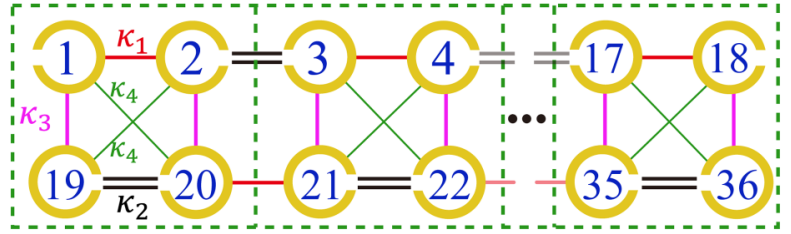

(c)

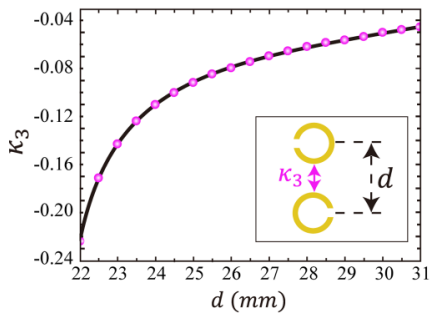

(d)

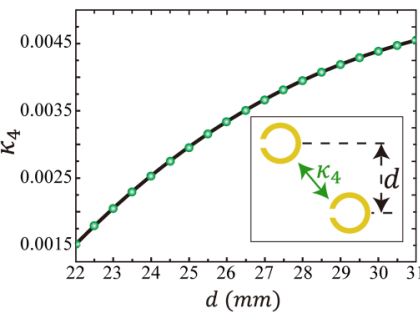

FIG. 3. (a) Photo of the Kitaev chain composed of two SRR chains. The sample is put on a 1-cm-thick foam plate and sandwiched between two aluminum covers. (b) Schematic model of the Kitaev chain. Specifically, the relative rotation angles of the slits of two resonators in the unit cell of the upper chain and lower chain are $0^{\circ}$ and $180^{\circ}$, respectively. The intrachain coupling coefficients in the upper and lower chains are denoted by $\kappa_{1}$ and $\kappa_{2}$, respectively. The interchain vertical coupling coefficient $\kappa_{3}$ (c) and diagonal coupling coefficient $\kappa_{4}$ (d) depend on the separation distance between the two SRR chains.

that in this SRR configuration, $\kappa_{4}$ would be much smaller than $\kappa_{3}$. Nevertheless, if we change SRRs to disks, $\kappa_{3}$ and $\kappa_{4}$ would be in the same order of magnitude. To confirm this point, in Figs. 3(c) and 3(d) we calculate the variance of $\kappa_{3}$ and $\kappa_{4}$ with $d$, respectively. From Fig. 3, we see that $\kappa_{3}$ and $\kappa_{1}\left(\right.$ or $\left.\kappa_{2}\right)$ are in the same order of magnitude while $\kappa_{4}$ is always much smaller than $\kappa_{3}$. As a result, $\kappa_{4}$ can be ignored and our structure can be taken as an effective Kitaev model in which the $\kappa_{1} \gg \kappa_{4}$ condition is satisfied [34].

Then, considering different values of $d$, we calculate the eigenvalue spectra of Kitaev chain and the topological phase diagrams for the Kitaev chains with different chain lengths shown in the Appendix B. In the experiment, the total number of the SRRs in the Kitaev chain is selected to be 36, as is shown in Fig. 3(a). The sample is put on a foam substrate with a permittivity of near 1 . Then, the sample sustained by the foam plate is put into a metallic planar waveguide in which two aluminum covers are separated by $30 \mathrm{~mm}$. Since the resonance frequency of SRR $(1.836 \mathrm{GHz})$ is below the cutoff frequency of waveguide $(5.0 \mathrm{GHz})$, the interaction between SRRs can only rely on the near-field coupling and the SRR chain can be treated by the tight-binding model. In 

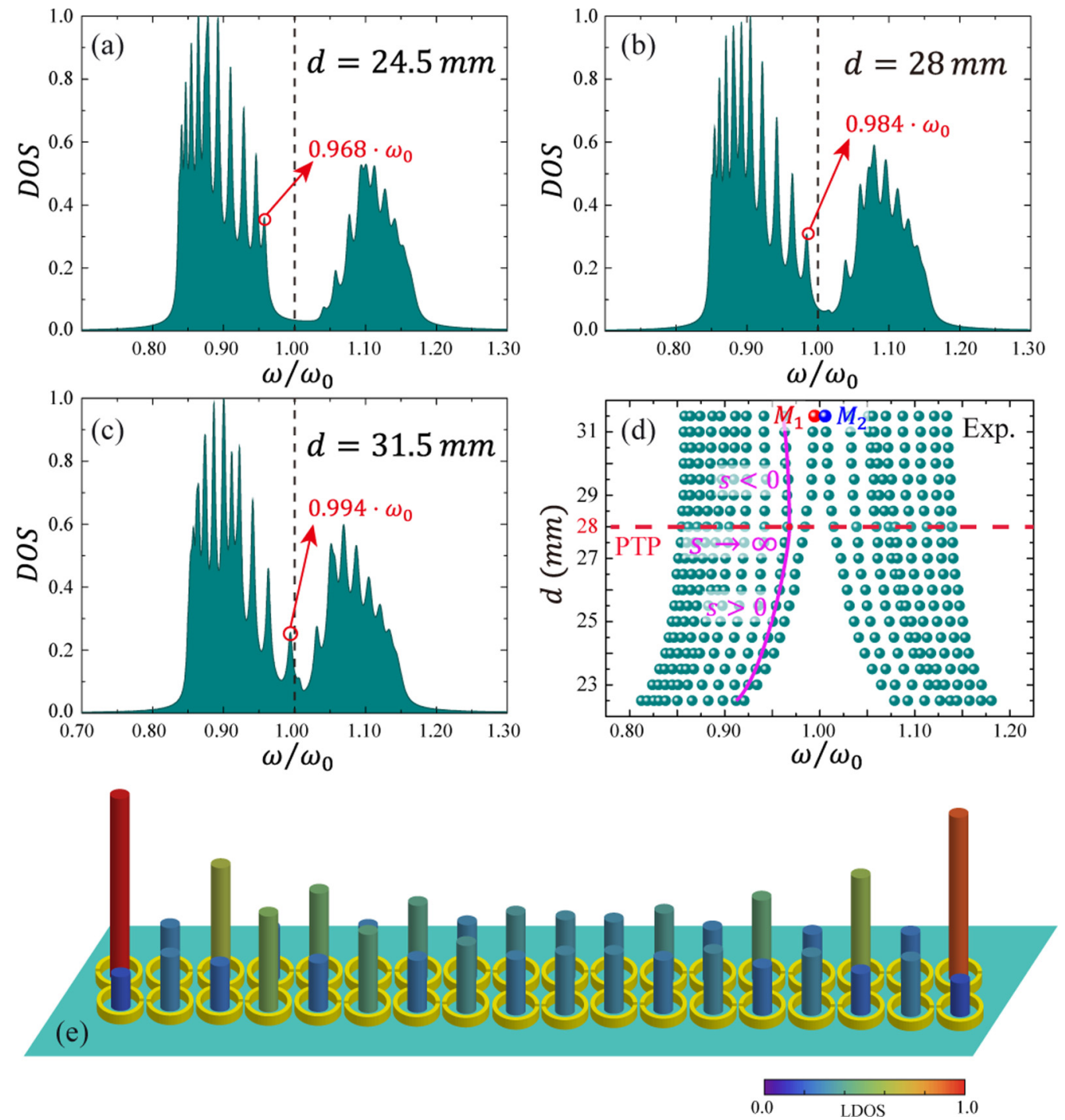

FIG. 4. (a) Measured normalized DOS spectrum of the topological trivial Kitaev chain with $d=24.5 \mathrm{~mm}$. Two bands are separated by a gap. (b) Same as (a), but this case corresponds to the PTP with $d=28 \mathrm{~mm}$. (c) Same as (a) and (b), but this case corresponds to the topological nontrivial Kitaev chain with $d=31.5 \mathrm{~mm}$. (d) Measured topological phase diagrams for the Kitaev chain with 36 identical SRRs. The fitted curve and the position of the PTP are marked by the solid purple line and red dashed line, respectively. (e) The normalized LDOS distribution of the topological bound state [marked by $M 1$ in (d)] in the Kitaev chain without disorder.

Fig. 3(a), the magnetic probe is a home-made loop antenna, which is connected to the vector network analyzer (Agilent PNA Network Analyzer N5222A). The distance between the probe and sample is $1 \mathrm{~mm}$. Based on the near-field detection method, the probe can measure the local density of states (LDOS) at each site of the Kitaev chain. Then, the DOS spectrum is obtained by averaging the LDOS spectra over all sites [25]. Considering Kitaev chains with three values of $d=$ $24.5 \mathrm{~mm}, d=28 \mathrm{~mm}$, and $d=31.5 \mathrm{~mm}$, the corresponding normalized DOS spectrum is shown in Figs. 4(a)-4(c), respectively. Because the loss of the system increases with the increase of frequency, the DOS spectrum has a higher peak value and a narrower linewidth at the low-frequency side, and a lower peak value and a wider linewidth at the highfrequency side $[55,56]$. With the increase of $d$, a topological transition will take place from the topological trivial phase [Fig. 4(a)] to the topological nontrivial phase [Fig. 4(c)]. To see clearly this phase transition, the evolution of the lowfrequency topological bound state is indicated by the red arrows in Figs. 4(a)-4(c). The peaks in the different DOS spectrum of each Kitaev chain are extracted as the modes indicated by the dots in Fig. 4(d). Specifically, it is to extract the frequency corresponding to each peak in a different DOS spectrum of each Kitaev chain and then it is plotted as a function of the distance. From the phase diagram Fig. 4(d), as the value of $d$ increases, the Kitaev chain changes from a topological trivial structure to a nontrivial one and topological bound states appear in the gap. Three states marking the evolution of the topological bound state in Figs. 4(a)-4(c) are also marked by red arrows as shown. In Fig. 4(d), we fit the $n / 2-1$ modes corresponding to the low-frequency gap-edge modes by a solid purple curve. This curve is cut off at a point in which the slope diverges. This cutoff point just corresponds to the position of the PTP, as is marked by 
(a)

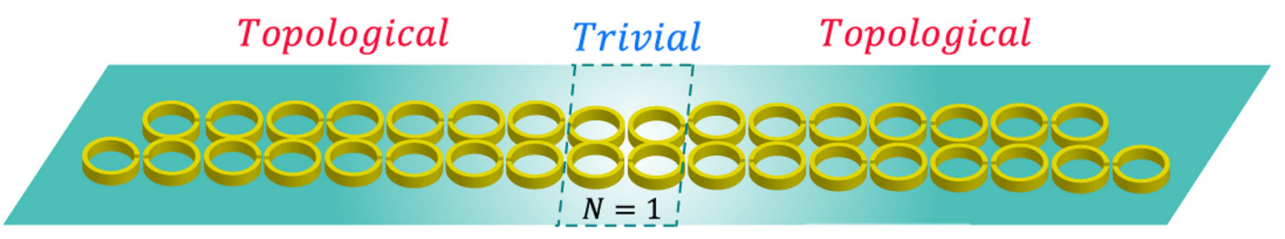

(b)

(c)
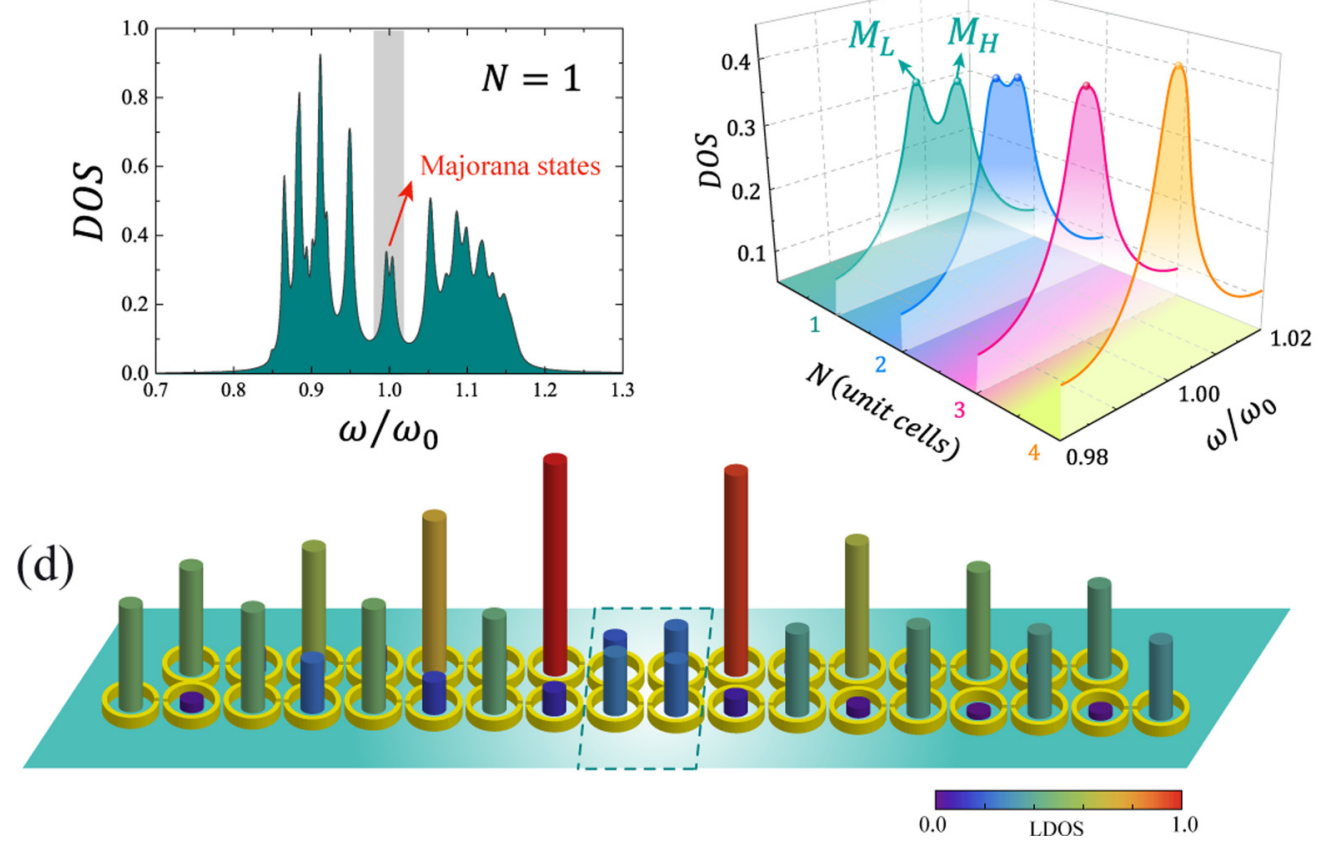

FIG. 5. (a) Schematic of the composite Kitaev chain, in which a trivial chain $(d=24.5 \mathrm{~mm})$ is sandwiched by two topological chains $(d=31.5 \mathrm{~mm})$. The coupling strength of two topological bound states can be tuned by changing the length of trivial chain. (b) Measured normalized DOS spectrum of the composite Kitaev chain. There are two topological bound states with different frequencies in the band gap, which are marked by the red arrow. (c) Similar to (b), but the enlarged DOS spectrum with increasing the length of inner trivial chain. (d) The normalized LDOS distribution of the topological bound state [marked by $M_{\mathrm{L}}$ in Fig. 5(c)] in the Kitaev chain.

the red dashed line $(d=28 \mathrm{~mm})$ in Fig. 4(b). There are some differences between the experimental phase diagram and the theoretical phase diagram (see more details in Appendix B), which is mainly caused by two factors. On the one hand, the coupling strength in the experiment is slightly less than that in the theoretical calculation, which leads to the deviation of the phase-transition point. On the other hand, the experimental phase diagram is not as clear as the theoretical phase diagram, which is mainly because the experimental mode is obtained by extracting the frequency of the peak from the DOS spectrum. The quality factor of some peaks in the DOS spectrum is not high enough, resulting in overlapping, so the data points are not as much as the theory, and the experimental results are not as clear as the theoretical results. Take the topological nontrivial phase $d=31.5 \mathrm{~mm}$ as an example; two topological bound states are marked by $M_{1}\left(\omega=0.994 \omega_{0}\right)$ and $M_{2}$ $\left(\omega=1.006 \omega_{0}\right)$, respectively. From the measured LDOS distribution in Fig. 4(e), we can see that the topological bound state is confined at two ends of the upper chain. The robust property of the topological bound state against disorders is shown in Appendix C. The above experiments show that the topological bound state in the topological nontrivial chain is confined at the two ends of the upper chain. Furthermore, the controllability of the topological bound states based on a reconfigurable quasiperiodic Hofstadter chain has also been theoretically studied, which is shown in Appendix D.

\section{COUPLING OF TOPOLOGICAL BOUND STATES}

In the above section, we have experimentally measured the phase diagram of Kitaev chain, observed the photonic topological bound states in the topological nontrivial phase, and theoretically studied the flexible distribution of topological bound states in a reconfigurable quasiperiodic chain. In this section, based on the measured phase diagram, we study the coupling between two topological bound states in a composite Kitaev chain. The schematic of the structure is shown in Fig. 5(a), which is composed of the topological chains $(d=$ $31.5 \mathrm{~mm})$ on both sides and the trivial chain $(d=24.5 \mathrm{~mm})$ in the middle. The unit-cell number of the trivial chain is 1 as shown in Fig. 5(a). In fact, the coupling strength of the topological bound states can be effectively tuned by changing the length of trivial chain. Specifically, in order to clearly see the coupling of topological bound states on both sides of the trivial chain, we remove two SRRs at both ends of the upper chain, as is shown in Fig. 5(a). When the unit-cell number 
of the trivial chain is $N=1$, the measured normalized DOS spectrum is shown in Fig. 5(b). The central part of the band gap is marked by a shadow region, and two topological bound states appear in the middle of the band gap [see Fig. 5(b)]. Figure 5(c) shows the enlarged DOS spectrum of a trivial chain of different lengths from $N=1$ to $N=4$. Considering the low-frequency topological bound state for $N=1$, which is marked by $M_{\mathrm{L}}$ in the enlarged DOS spectrum Fig. 5(c), we measured the normalized LDOS distribution in Fig. 5(d). It is obvious that there is strong localization at the two interfaces between the trivial chain and the topological chains, which is the result of the coupling of the two topological main states. With increasing the length of the trivial chain, we find that the two topological bound states $\left(M_{\mathrm{L}}\right.$ and $\left.M_{\mathrm{H}}\right)$ are approaching gradually and overlap at the degenerate point, as is shown in Fig. 5(c). The coupling between topological bound states may be used to explore some practical applications, such as switching and sensor with topological protection. Moreover, in order to study the exceptional point (EP) physics based on the non-Hermitian Kitaev chain, we theoretically propose a composite Kitaev chain satisfying $P T$ symmetry. The EP formed by topological bound states and the related application in a topological sensor are studied theoretically (see more details in Appendix E).

\section{CONCLUSION}

A double chain composed of SRRs provides us a good platform to study the complex topological model and reveal abundant physical mechanism. By avoiding the diagonal coupling and delicately tuning the vertical coupling of the two chains, we experimentally study the transition process from a topological trivial phase to a nontrivial phase in an effective Kitaev model. Based on the measured topological phase diagram we also find that the coupling of topological bound states can be flexibly tuned by changing the length of the trivial chain. In addition, we theoretically reveal that the spatial confinement position of the topological bound state can be changed flexibly after considering the quasiperiodic modulation of the Kitaev chain. Finally, we propose to use a $P T$ symmetric composite Kitaev chain to construct a high sensitivity sensor with topological protection characteristics. Our results not only reveal the establishing process of photonic topological bound states and EPs, but also may provide a versatile platform to study another important topological class of systems with complex coupling distributions and potential applications.

\section{ACKNOWLEDGMENTS}

The authors would like to thank Prof. $\mathrm{X}$. Hu from the International Center for Materials Nanoarchitectonics (WPI-MANA) of Japan for fruitful discussions. This work was sponsored by the National Key Research Program of China (Grant No. 2016YFA0301101), the National Natural Science Foundation of China (NSFC) (Grants No. 12004284, No. 11935010, No. 11775159, and No. 61621001), the Natural Science Foundation of Shanghai (Grants No. 18ZR1442800 and No. 18JC1410900), by the China Postdoc- (a)

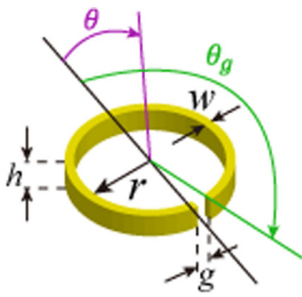

(b)

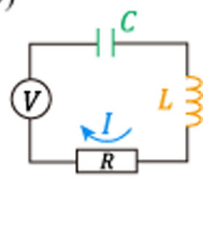

(c)

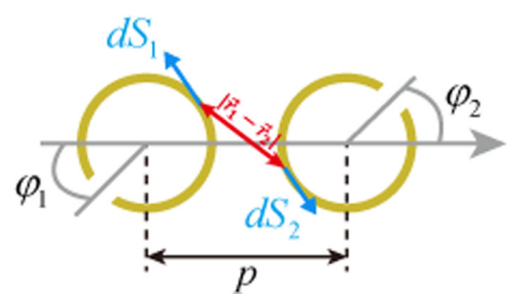

FIG. 6. (a) The schematic of the single SRR. (b) Equivalent circuit of the single SRR. (c) Sketch of a pair of arbitrarily rotated split rings.

toral Science Foundation (Grants No. 2019TQ0232 and No. 2019M661605), by the Shanghai Super Postdoctoral Incentive Program, and by the Opening Project of the Shanghai Key Laboratory of Special Artificial Microstructure Materials and Technology.

\section{APPENDIX A: DEPENDENCE OF THE COUPLING COEFFICIENT ON THE RELATIVE ROTATION ANGLE AND THE SEPARATING DISTANCE}

The SRRs studied in this paper are constructed from a copper ring with a single gap, as is shown in Fig. 6(a). The corresponding equivalent circuit is shown in Fig. 6(b). $L, C$, and $R$ represent the inductance, capacitance and resistance, respectively. The coupling between two arbitrarily rotated SRRs consists of magnetic and electric coupling [57]. In Fig. 6(c), the rotating angles of the rings are $\varphi_{1}$ and $\varphi_{2}$, respectively. The type of dominant coupling and the coupling strength can be controlled by tuning the relevant angle between two resonators. So, SRRs provide us a good platform to study some interesting and complex physical models that are difficult to implement in condensed state systems.

The total coupling between two SRRs is $\kappa=\kappa_{E}+\kappa_{H}$, where $\kappa_{E}$ and $\kappa_{H}$ are the electric and magnetic coupling, respectively. It can be determined with the aid of the charge and current densities in the split rings. The charge distribution in the split ring is given as [57-59]

$$
\rho_{L n}(\theta)=\left(\tan \frac{\theta}{2}\right) / 2 \ln \left(\frac{g}{4 r}\right) \quad\left(\theta<\theta_{g}\right),
$$

where $\pm \theta_{g}$ are the coordinates of the gap [see Fig. 6(a)]. And the normalized current is of the form

$$
I_{n}(\theta)=\ln \left(\frac{\cos \frac{\theta_{g}}{2}}{\cos \frac{\theta}{2}}\right) \ln ^{-1}\left(\cos \frac{\theta_{g}}{2}\right) .
$$

The total magnetic energy can be expressed as

$$
W=\frac{\mu_{0}}{4 \pi} \iint \frac{\vec{J}_{1}\left(\vec{r}_{1}\right) \cdot \vec{J}_{2}\left(\vec{r}_{2}\right) e^{i k\left|\vec{r}_{1}-\vec{r}_{2}\right|}}{\left|\vec{r}_{1}-\vec{r}_{2}\right|} d \tau_{1} d \tau_{2},
$$



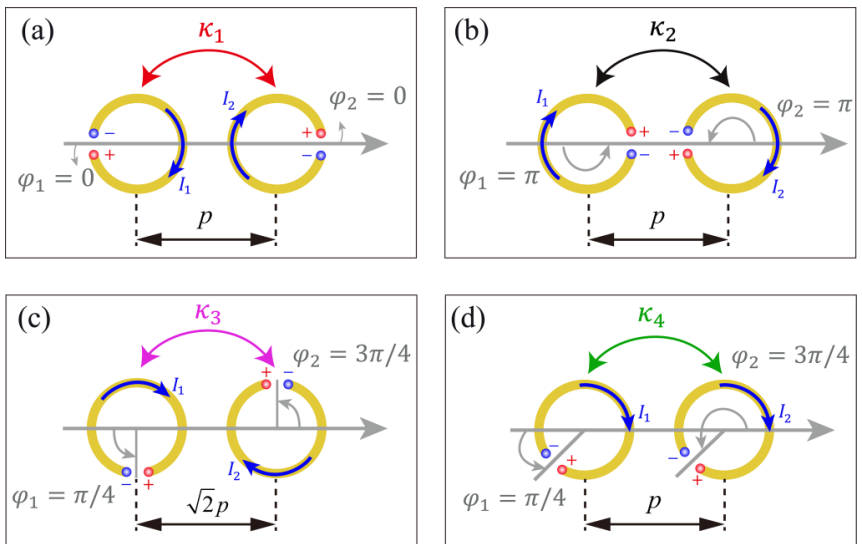

FIG. 7. Four kinds of coupling in Kitaev chain composed of SRRs.

where $\vec{J}_{l}(l=1,2)$ and $\vec{A}_{l}(l=1,2)$ denote the volume current density and magnetic vector potential in SRRs respectively. $\mu_{0}$ is the permeability in free space. $d \tau$ is a volume element. Because $\vec{J}_{l}(l=1,2)=w h \vec{h}_{l}(l=1,2)$, Eq. (A3) can be written as the function of line current density,

$$
W_{M}=\frac{\mu_{0}}{4 \pi} \iint \frac{\vec{I}_{1}\left(\vec{r}_{1}\right) \cdot \vec{I}_{2}\left(\vec{r}_{2}\right) e^{i k\left|\vec{r}_{1}-\vec{r}_{2}\right|}}{\left|\vec{r}_{1}-\vec{r}_{2}\right|} d s_{1} d s_{2},
$$

where $d s$ is a line element.

The mutual inductance $M=W_{M} / I_{1} I_{2}$ can be obtained from the equation [57]

$$
M=\frac{\mu_{0}}{4 \pi} \iint \frac{\vec{I}_{n 1}\left(\vec{r}_{1}\right) \cdot \vec{I}_{n 2}\left(\vec{r}_{2}\right) e^{i k\left|\vec{r}_{1}-\vec{r}_{2}\right|}}{\left|\vec{r}_{1}-\vec{r}_{2}\right|} d s_{1} d s_{2} .
$$

Similarly, the total electric energy can be expressed as

$$
W_{E}=\frac{1}{4 \pi \varepsilon_{0}} \iint \frac{\rho_{1}\left(\vec{r}_{1}\right) \rho_{2}\left(\vec{r}_{2}\right) e^{i k\left|\vec{r}_{1}-\vec{r}_{2}\right|}}{\left|\vec{r}_{1}-\vec{r}_{2}\right|} d \tau_{1} d \tau_{2},
$$

where $\rho_{l}(l=1,2)$ means the corresponding normalized line charges in SRRs and $\varepsilon_{0}$ is the free-space permittivity. So the mutual capacitance $K^{-1}=-W_{E} / Q_{1} Q_{2}\left[Q_{l}(l=1,2)\right.$ is the total charge on one half of the SRR] can be obtained as $[57,58]$

$$
K^{-1}=\frac{1}{4 \pi \varepsilon_{0}} \iint \frac{\rho_{L n 1}\left(\vec{r}_{1}\right) \rho_{L n 2}\left(\vec{r}_{2}\right) e^{i k\left|\vec{r}_{1}-\vec{r}_{2}\right|}}{\left|\vec{r}_{1}-\vec{r}_{2}\right|} d \tau_{1} d \tau_{2},
$$

where $\rho_{\text {Lnl }}(l=1,2)$ means the corresponding normalized line charges.
The approximate values of the self-inductance and selfcapacitance are [57]

$$
L=\mu_{0} r\left(\ln \frac{8 r}{h+w}-\frac{1}{2}\right)
$$

and

$$
C=\varepsilon_{0}\left[\frac{(w+g)(h+g)}{g}+\frac{2(h+w)}{\pi} \ln \left(\frac{4 r}{g}\right)\right] .
$$

So, the total coupling is $\kappa=\kappa_{E}+\kappa_{H}$ can be determined by Eqs. (A4) and (A7)-(A9) with

$$
\kappa_{H}=\frac{2 M}{L}, \quad \kappa_{E}=-\frac{2 C}{K} .
$$

Based on Eq. (A10), four kinds of coupling between two SRRs in a Kitaev chain can be obtained and the dominant coupling cases can be seen in Fig. 7.

\section{APPENDIX B: TOPOLOGICAL PHASE DIAGRAMS OF KITAEV CHAINS WITH DIFFERENT LENGTHS}

For a finite-periodic Kitaev chain with different $d$, the property of the band gaps is quite different. In the region that the values of $d$ are small, there is no topological bound state in the gap and the structure is in the topological trivial phase. In this region, the $n / 2$ modes correspond to the lowfrequency gap-edge modes while the $n / 2-1$ modes are in the band. In contrast, in the region that the values of $d$ are large, topological bound states appear in the gap and the structure is in the topological nontrivial phase. In this region, the $n / 2$ modes correspond to the topological bound states while the $n / 2-1$ modes correspond to the low-frequency gap-edge modes. From Fig. 8, we see that the boundary between nontrivial and trivial phases becomes unclear as the number of SRRs decreases. To determine the position of PTP, we fit the positions of the $n / 2-1$ modes that correspond to the low-frequency gap-edge modes by solid purple curves. The expressions of these three fitting curves are as follows:

$$
\begin{aligned}
\omega_{36}(d)= & -3.116 \times 10^{-7} d^{8}+6.848 \times 10^{-5} d^{7}-6.573 \\
& \times 10^{-3} d^{6}+0.36 d^{5}-12.3 d^{4}+268.8 d^{3} \\
& -3663 d^{2}+2.849 \times 10^{4} d-9.68 \times 10^{4},
\end{aligned}
$$

(a)

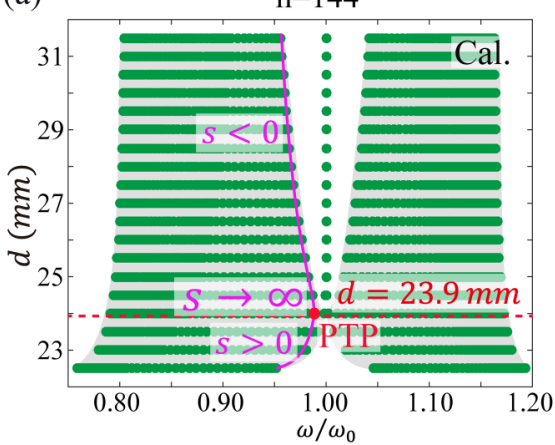

(b)

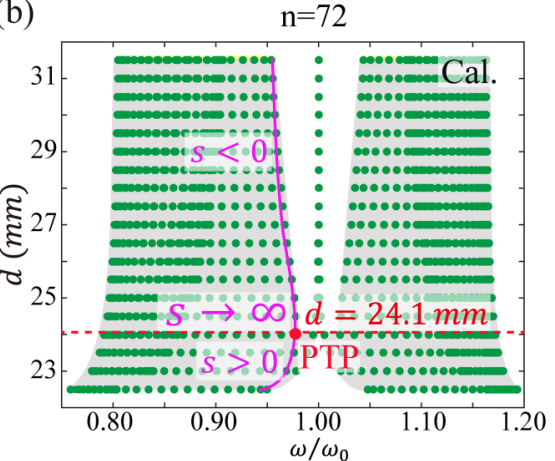

(c)

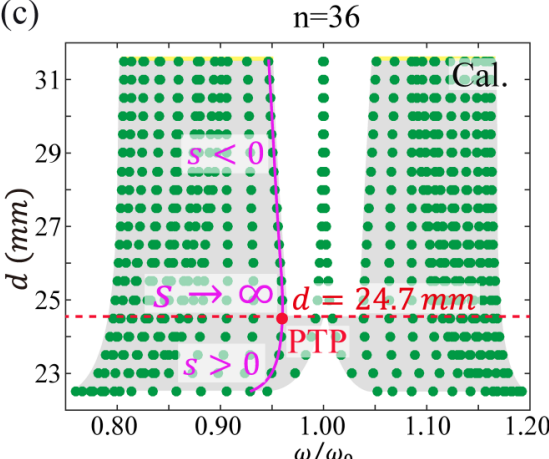

FIG. 8. Calculated topological phase diagrams for the Kitaev chains with 144 (a), 72 (b), and 36 (c) identical SRRs, respectively. 
(a)

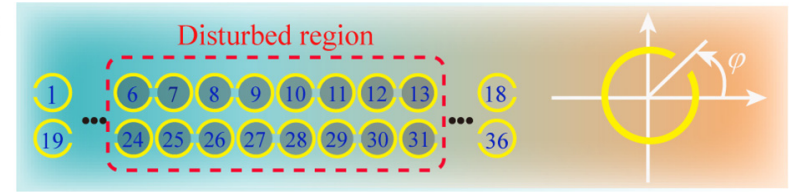

(b)

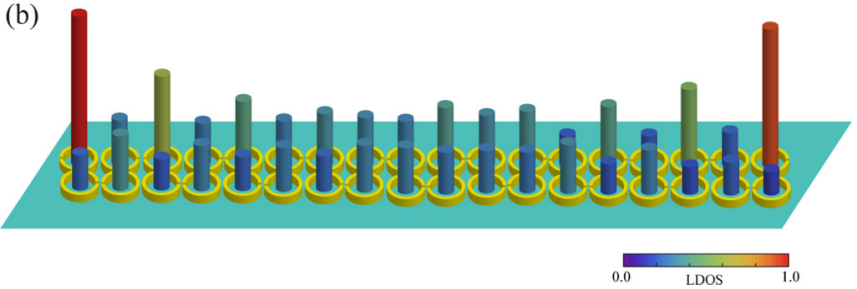

FIG. 9. (a) The topological Kitaev chain after disorder is introduced into the 16 SRRs indicated by the grey circles. The right inset shows the rotating angle for the disorder. (b) The normalized LDOS distribution of the topological bound state [marked by $M 1$ in Fig. 4(d)] in the Kitaev chain without disorder. (c) Similar to (b), but with disorder.

$$
\begin{aligned}
\omega_{72}(d)= & -2.811 \times 10^{-7} d^{8}+6.18 \times 10^{-5} d^{7}-5.935 \\
& \times 10^{-3} d^{6}+0.3253 d^{5}-11.31 d^{4}+243.2 d^{3} \\
& -3319 d^{2}+2.585 \times 10^{4} d-8.797 \times 10^{4}, \\
\omega_{144}(d)= & -8.153 \times 10^{-7} d^{8}+1.833 \times 10^{-5} d^{7}-1.802 \\
& \times 10^{-3} d^{6}+0.1012 d^{5}-3.55 d^{4}+79.68 d^{3} \\
& -1117 d^{2}+8951 \times 10^{4} d-3.316 \times 10^{4} . \quad(\mathrm{B} 1)
\end{aligned}
$$

These curves are cut off at the points in which the slope diverges, $S \rightarrow \infty$, as shown in Fig. 8. These cutoff points just correspond to the positions of PTPs. In three finite-size structures with $n=144,72$, and 36, PTPs occur at $d=23.9$, 24.1, and $24.7 \mathrm{~mm}$, respectively, as marked by red dashed lines in Figs. 8(a)-8(c). Comparing Fig. 8(c) with Fig. 4(d), we see that the measured phase diagram in Fig. 4(d) is consistent with the calculated one in Fig. 8(c). The small deviation between the experimental result and calculation is caused by the tolerance in the fabrication and measurement.

\section{APPENDIX C: ROBUST PROPERTY OF PROTECTED TOPOLOGICAL BOUND STATES AGAINST DISORDERS}

In Fig. 9(a), perturbation is introduced by rotating the interior of the 16 SRRs indicated by the grey circles. The detail of rotation is illustrated in the right inset of Fig. 9(a). The rotating disorder is introduced by randomly rotating the SRRs from $-5^{\circ}$ to $5^{\circ}$ into the interior of the rings. For comparison, at first, we see normal Kitaev chains without disorder. Take the topological nontrivial phase $d=31.5 \mathrm{~mm}$ as an example; the normalized LDOS distribution of the topological bound state which corresponds to the $M 1$ state in Fig. 4(d) is shown in Fig. 9(b). We see that the topological bound state is confined at two ends of the upper chain. Then we add the disorder indicated in Fig. 9(a) into the Kitaev chain. From Fig. 9(c), it is seen that the topological bound state is still confined at the two ends of the upper chain, which is hardly influenced (a)

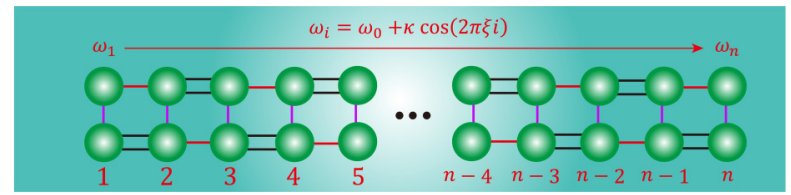

(b)

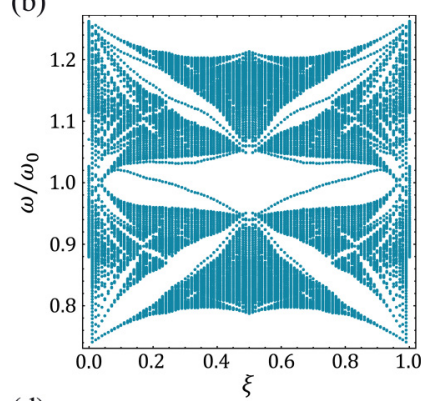

(c)
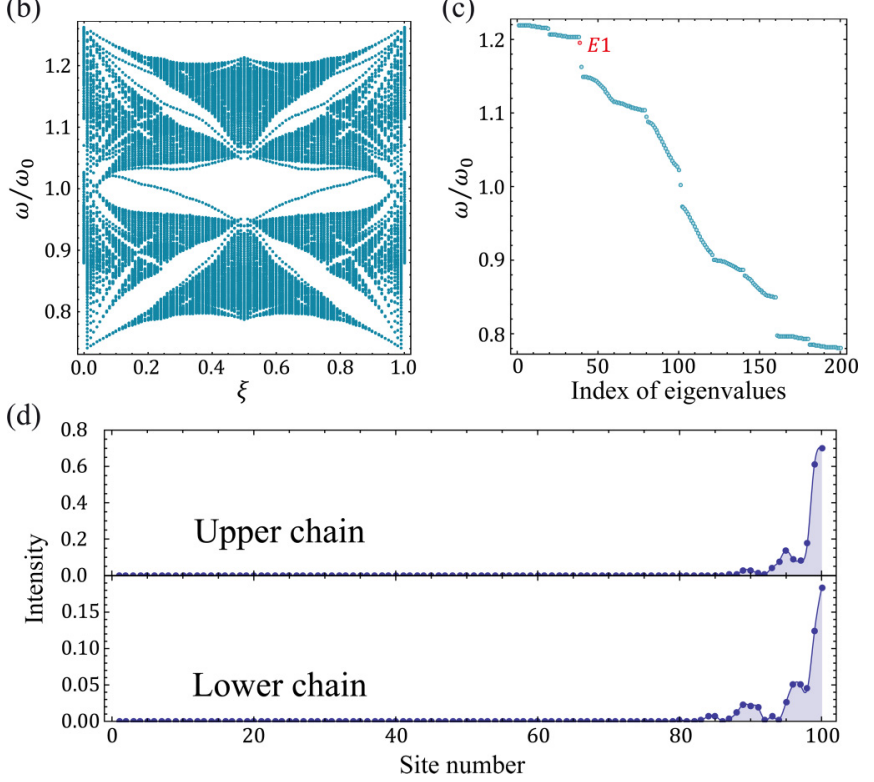

FIG. 10. (a) Schematic of the Hofstadter model in reconfigurable quasiperiodic chain. In this tight-binding model, the quasiperiodic modulation is realized by tuning the interchain coupling. (b) The phase diagram of the finite-size Hofstadter chain as a function of the parameter $\xi$ when. (c) The eigenvalues spectra of the Hofstadter chain in (a) when $\xi=0.2$. (d) The intensity distributions of edge state $E 1$ in (c). The edge state is localized at the right end of the upper and lower chains.

by the disorder. The topological bound states that are robust against perturbations may have some potential applications in the energy transfer.

\section{APPENDIX D: HOFSTADTER CHAIN WITH QUASIPERIODIC INTERCHAIN COUPLING}

In this Appendix, we show that the distribution of the topological bound state in a Kitaev chain can be flexibly controlled in a reconfigurable quasiperiodic Hofstadter chain [60]. The topological chain with finite resonators in a quasiperiodic arrangement is controlled by tuning the on-site frequency as [61]

$$
\omega_{i}=\omega_{0}+\kappa \cos (2 \pi \xi i),
$$

where $\omega_{0}=1.836 \mathrm{GHz}$ is the unperturbed on-site resonance frequency of SRR. $\omega_{i}$ is interpreted as the $i$ th on-site resonance frequency, as is shown in Fig. 10(a). $\kappa=\kappa_{1}+\kappa_{2}$ is the coefficient that controls the strength of the modulation. This variation is of particular interest in the context of localization physics. Considering a finite chain with $n=100$, we vary the value of $\xi$ from 0 to 1 and obtain the phase diagram, which is shown in Fig. 10(b). This phase diagram shows that the spectrum is fractal. One can see that there is a fractal network of spectral gaps, which is known as Hofstadter's butterfly [60]. 
(a)

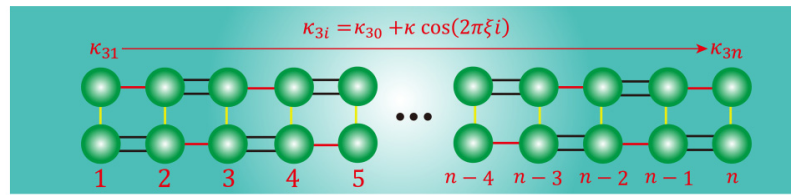

(b)

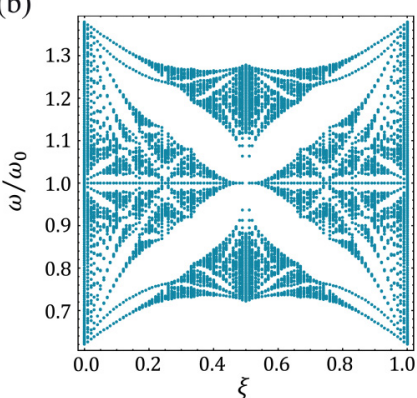

(c)

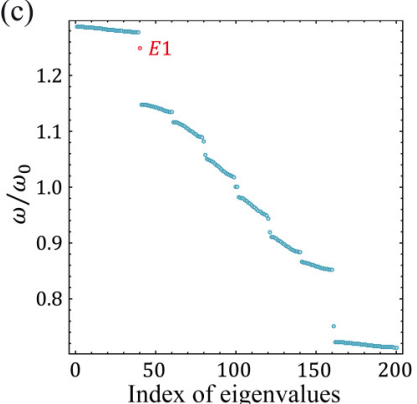

(d)

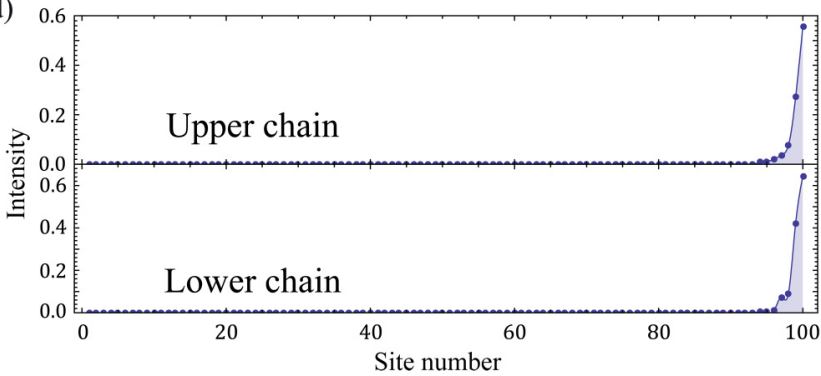

FIG. 11. (a) Schematic of the Hofstadter model in reconfigurable quasiperiodic chain. In this tight-binding model, the quasiperiodic modulation is realized by tuning the on-site frequency. (b) The phase diagram of the finite-size Hofstadter chain as a function of the parameter $\xi$. (c) The eigenvalues spectra of the Hofstadter chain in (a) when $\xi=0.2$. (d) The intensity distributions of edge state $E 1$ in (c). The edge state is localized at the right end of the upper and lower chains.

In the microwave regime, the quasiperiodic modulation of on-site resonance frequency of SRR can be actively controlled [62]. By adding a variable capacitance diode into the split of SRR, the on-site frequency can be flexibly tuned by an external voltage $[63,64]$. When considering the quasiperiodic modulation of the interchain coupling, we can also get a clear spectral butterfly. Take $\xi=0.2$ as an example; the eigenvalues spectra of the Hofstadter chain is shown in Fig. 10(c). The edge states can be identified by the discrete modes in the band gap. In contrast to the edge state of a periodic Kitaev chain in which the field amplitude is localized at the two ends of the upper chain, the edge state E1 in Fig. 10(c) is localized at the right end of the upper and lower chains, as is shown in Fig. 10(d). It can be seen from Fig. 10 that the spatial distribution of topological bound state can be flexibly controlled by considering quasiperiodic modulation into a Kitaev chain. It is worth noting that although we only show the calculation results here, the experiment of manipulating the topological bound states by applying voltage is expected to be carried out $[63,64]$. In addition, by proper arrangement of the SRRs, other interesting topological physics can be studied in the future, such as the high-order topological structures [65-67].

Considering the quasiperiodic modulation of the interchain coupling [Fig. 11(a)], the spectral butterfly is shown (a)

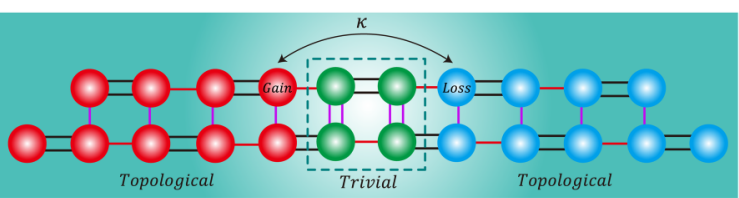

(b)

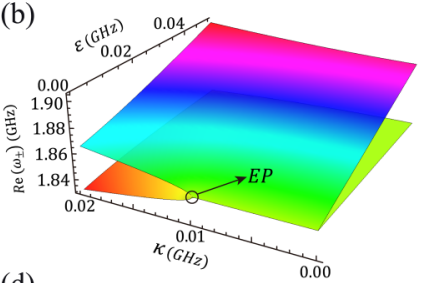

(d)
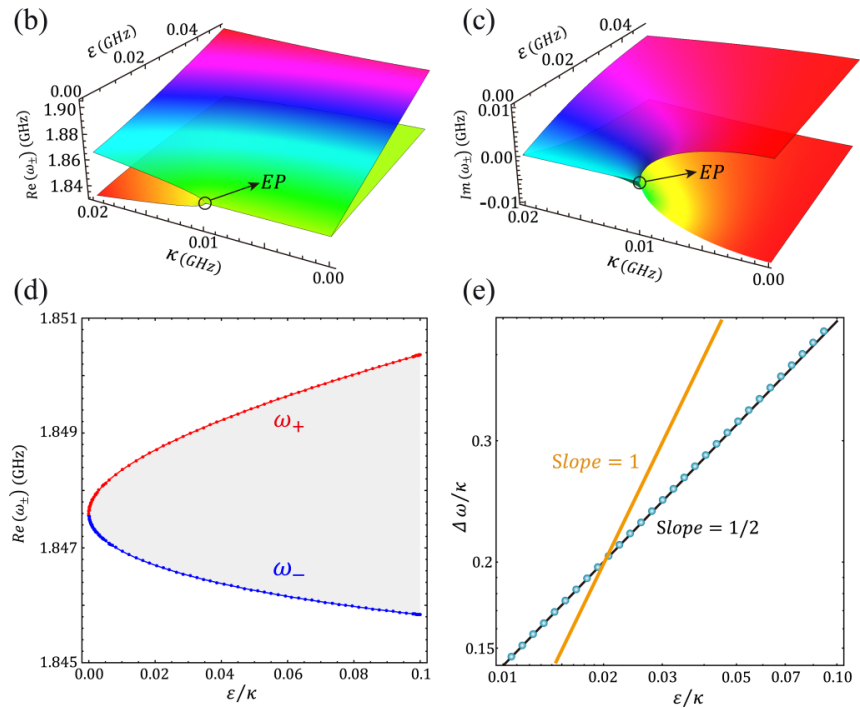

FIG. 12. (a) Schematic of the composite Kitaev chain with PT symmetry, in which the left chain and right topological chains with gain and loss respectively. The real part (b) and imaginary part (c) of the eigenfrequencies of two coupled edge states as a function of the coupling strength $\kappa$ and the frequency detuning $\varepsilon$. (d) For EP, the real part of the eigenfrequencies, as a function of the normalized frequency detuning $\varepsilon / \kappa$. (e) Dependence of the normalized frequency splitting $\Delta \omega / \kappa$ on the normalized frequency detuning $\varepsilon / \kappa$. The results on a logarithmic scale as shown. The reference yellow and black lines with the slope $=1$ and $1 / 2$ are displayed by $\Delta \omega / \kappa=\varepsilon / \kappa$ and $\Delta \omega / \kappa=\sqrt{\varepsilon} / \kappa$ respectively.

in Fig. 11(b). Similar to the quasiperiodic modulation of the on-site frequency in Fig. 10, we take $\xi=0.2$ as an example. The eigenvalue spectra of the Hofstadter chain in Fig. 11(a) is shown in Fig. 11(c). The edge states can be determined by the discrete modes in the band gap and the edge state $E 1$ in Fig. 11(c) is localized at the right end of the upper and lower chains, as is shown in Fig. 11(d). The results of Figs. 10 and 11 are consistent.

\section{APPENDIX E: TOPOLOGICAL BOUND STATE IN A NON-HERMITIAN KITAEV CHAIN}

Exceptional points (EPs) in optics and photonics have attracted great attention for many applications, such as sensors, multimode lasers, and dynamic wireless energy transmission [68-71]. Importantly, although EPs provide great advantages on realizing sensors [72,73], it has been pointed out that EP sensors based on two coupled rings with balanced gain and loss are very sensitive to fabrication errors and the disorder of structures [74]. So, it is urgent to realize robust EPs in designing new sensors. Recently, simultaneous observation of a topological edge state and an EP in a non-Hermitian system has attracted great attention. The possibility of 
obtaining the abundant non-Hermitian topological properties in optical platforms has a profound influence on optical science and information communication [75]. The EP based on the coupling of topological bound states can solve the abovementioned problem very well. On the one hand, similar to the systems of coupled resonators, an EP formed by two interface states is sensitive to the interface perturbations [71,72]. On the other hand, the EP realized in the topological systems inherits the robustness of the topological bound states and it will be immune to fluctuations and disorders within the structure. Therefore, we study the coupling of topological bound states by combining the topological chain and the trivial chain, which provides a good platform for further research on applications related to the EPs with topological protection, such as ultrasensitive sensors, switching, and energy transfer [74-80].

Different from the structure in Fig. 5, appropriate loss and gain are added to the left and right topological chains respectively. The schematic of the composite Kitaev chain with $P T$ symmetry is shown in Fig. 12(a). Within the context of coupled mode theory, the coupling of two topological bound states can be described by the effective Hamiltonian

$$
i \frac{d}{d t}\left[\begin{array}{l}
a \\
b
\end{array}\right]=H_{M}\left[\begin{array}{l}
a \\
b
\end{array}\right], \quad H_{M}=\left[\begin{array}{cc}
\omega_{0}+i g-i \Gamma & \kappa \\
\kappa & \omega_{0}-i \gamma-i \Gamma
\end{array}\right],
$$

where $a$ and $b$ are the field amplitudes of the topological bound states on the left and right sides, respectively. $\Gamma$ is the loss rate of topological bound states including intrinsic absorption $\Gamma_{1}=0.00148 \mathrm{GHz}$ and radiation loss $\Gamma_{2}=$ $0.00595 \mathrm{GHz}$, which can be obtained by the measured spectrum in Fig. 5. $\kappa$ describes the near-field interaction between two topological bound states. We assume $g=0.01743 \mathrm{GHz}$ and $\gamma=0.00257 \mathrm{GHz}$ are the gain and loss added in the left and right states respectively, which is subjected to the symmetry condition $i g-i \Gamma=i \gamma+i \Gamma$. It is worth mentioning that it is feasible to add controllable gain into SRR in the microwave regime $[81,82]$. The eigenvalues of
Eq. (E1) are

$$
\begin{aligned}
& \omega_{1-}=\frac{1}{2}\left(i g-i \gamma-2 i \Gamma-\sqrt{-(g+\gamma)^{2}+4 \kappa^{2}}+2 \omega_{0}\right), \\
& \omega_{1+}=\frac{1}{2}\left(i g-i \gamma-2 i \Gamma+\sqrt{-(g+\gamma)^{2}+4 \kappa^{2}}+2 \omega_{0}\right) .
\end{aligned}
$$

When $\kappa=0.01 \mathrm{GHz}$, two eigenstates coalesce, $\omega_{1-}=$ $\omega_{1+}$, which corresponds to the EP. EP is a non-Hermitian degeneracy that can enable intriguing applications such as enhanced sensing [72,73]. When a small perturbation $\varepsilon$ affects only the SRRs on the left topological Kitaev chain, the effective Hamiltonian can be written as

$$
H_{M P}=\left[\begin{array}{cc}
\omega_{0}+i g-i \Gamma+\varepsilon & \kappa \\
\kappa & \omega_{0}-i \gamma-i \Gamma
\end{array}\right] .
$$

The eigenvalues of Eq. (E3) are

$$
\begin{aligned}
& \omega_{-}=\frac{1}{2}\left(\varepsilon+i g-i \gamma-2 i \Gamma-\sqrt{[\varepsilon+i(g+\gamma)]^{2}+4 \kappa^{2}}+2 \omega_{0}\right), \\
& \omega_{+}=\frac{1}{2}\left(\varepsilon+i g-i \gamma-2 i \Gamma+\sqrt{[\varepsilon+i(g+\gamma)]^{2}+4 \kappa^{2}}+2 \omega_{0}\right) .
\end{aligned}
$$

The real and imaginary eigenfrequencies dependent on the coupling strength $\kappa$ and the frequency detuning $\varepsilon$ are shown in Figs. 12(b) and 12(c). The EP for the system without perturbation $(\varepsilon=0)$ is marked by the black arrows. To see the enhanced sensing for EP, which is realized by two topological bound states, we assume the small detuning $\varepsilon / \kappa \in$ $(0,0.1)$. The real part of the eigenfrequencies $\omega_{ \pm}$is shown in Fig. 12(d). The frequency splitting $\Delta \omega=\operatorname{Re}\left(\omega_{+}-\omega_{-}\right)$will increase as the perturbation increases in Fig. 12(d). The logarithmic plot of the relation between $\Delta \omega / \kappa$ and $\varepsilon / \kappa$ is shown by the green dotted line in Fig. 12(e). The reference yellow and black lines with the slope 1 and $1 / 2$ are displayed by $\Delta \omega / \kappa=\varepsilon / \kappa$ and $\Delta \omega / \kappa=\sqrt{\varepsilon} / \kappa$, respectively. The EP sensor composed of topological bound states exhibits a slope of $1 / 2$ for a small perturbation, confirming the square-root behavior. The different kind of sensor that combines the high sensitivity of EP and topological protection of edge states based on the non-Hermitian topological system is feasible in experiment and its potential application value is worth further study.
[1] M. Z. Hasan and C. L. Kane, Colloquium: Topological insulators, Rev. Mod. Phys. 82, 3045 (2010).

[2] X. L. Qi and S. C. Zhang, Topological insulators and superconductors, Rev. Mod. Phys. 83, 1057 (2011).

[3] L. Lu, J. D. Joannopoulos, and M. Soljačić, Topological photonics, Nat. Photon. 8, 821 (2014).

[4] T. Ozawa, H. M. Price, A. Amo, N. Goldman, M. Hafezi, L. Lu, M. Rechtsman, D. Schuster, J. Simon, O. Zilberberg, and I. Carusotto, Topological photonics, Rev. Mod. Phys. 91, 015006 (2019).

[5] Z. Wang, Y. D. Chong, J. D. Joannopoulos, and M. Soljačić, Observation of unidirectional backscattering-immune topological electromagnetic states, Nature (London) 461, 772 (2009).

[6] M. Hafezi, E. A. Demler, M. D. Lukin, and J. M. Taylor, Robust optical delay lines with topological protection, Nat. Phys. 7, 907 (2011).
[7] K. Fang, Z. Yu, and S. Fan, Realizing effective magnetic field for photons by controlling the phase of dynamic modulation, Nat. Photon. 6, 782 (2012).

[8] G. Q. Liang and Y. D. Chong, Optical Resonator Analog of a Two-Dimensional Topological Insulator, Phys. Rev. Lett. 110, 203904 (2013).

[9] A. B. Khanikaev, S. H. Mousavi, W.-K. Tse, M. Kargarian, A. H. MacDonald, and G. Shvets, Photonic topological insulators, Nat. Mater. 12, 233 (2013).

[10] W. J. Chen, S. J. Jiang, X. D. Chen, B. C. Zhu, L. Zhou, J W. Dong, and C. T. Chan, Experimental realization of photonic topological insulator in a uniaxial metacrystal waveguide, Nat. Commun. 5, 5782 (2014).

[11] L. H. Wu and X. Hu, Scheme for Achieving a Topological Photonic Crystal by Using Dielectric Material, Phys. Rev. Lett. 114, 223901 (2015). 
[12] Y. T. Yang, Y. F. Xu, T. Xu, H. X. Wang, J. H. Jiang, X. Hu, and Z. H. Hang, Visualization of a Unidirectional Electromagnetic Waveguide Using Topological Photonic Crystals Made of Dielectric Materials, Phys. Rev. Lett. 120, 217401 (2018).

[13] Y. Li, Y. Sun, W. W. Zhu, Z. W. Guo, J. Jiang, T. Kariyado, H. Chen, and X. Hu, Topological LC-circuits based on microstrips and observation of electromagnetic modes with orbital angular momentum, Nat. Commun. 9, 4598 (2018).

[14] M. C. Rechtsman, J. M. Zeuner, Y. Rlotnik, Y. Lumer, D. Podolsky, F. Dreisow, S. Nolte, M. Segev, and A. Szameit, Photonic Floquet topological insulators, Nature (London) 496, 196 (2013)

[15] B. Yang, Q. H. Guo, B. Tremain, R. J. Liu, L. E. Barr, Q. H. Yan, W. L. Gao, H. C. Liu, Y. J. Xiang, J. Chen, C. Fang, A. Hibbins, L. Lu, and S. Zhang, Ideal Weyl points and helicoid surface states in artificial photonic crystal structures, Science 359, 1013 (2018).

[16] M. A. Bandres, S. Wittek, G. Harari, M. Parto, J. Ren, M. Segev, D. N. Christodoulides, and M. Khajavikhan, Topological insulator laser: Experiments, Science 359, eaar4005 (2018).

[17] S. Mittal, E. A. Goldschmidt, and M. Hafezi, A topological source of quantum light, Nature (London) 561, 502 (2018).

[18] S. Barik, A. Karasahin, C. Flower, T. Cai, H. Miyake, W. De Gottard, M. Hafezi, and E. Waks, A topological quantum optics interface, Science 359, 666 (2018).

[19] Y. Yang, Z. Gao, H. Xue, L. Zhang, M. He, Z. Yang, R. Singh, Y. Chong, B. Zhang, and H. Chen, Realization of a threedimensional photonic topological insulator, Nature (London) $\mathbf{5 6 5}, 622$ (2019).

[20] Q. S. Huang, Z. W. Guo, J. T. Feng, C. Y. Yu, H. T. Jiang, Z. Zhang, Z. S. Wang, and H. Chen, Observation of a topological edge state in the X-ray band, Laser Photon. Rev. 13, 1800339 (2019).

[21] W. P. Su, J. R. Schrieffer, and A. J. Heeger, Solitons in Polyacetylene, Phys. Rev. Lett. 42, 1698 (1979).

[22] N. Malkova, I. Hromada, X. Wang, G. Bryant, and Z. Chen, Observation of optical Shockley-like surface states in photonic superlattices, Opt. Lett. 34, 1633 (2009).

[23] Y. X. Xiao, G. C. Ma, Z. Q. Zhang, and C. T. Chan, Topological Subspace-Induced Bound State in the Continuum, Phys. Rev. Lett. 118, 166803 (2017).

[24] D. A. Dobrykh, A. V. Yulin, A. P. Slobozhanyuk, A. N. Poddubny, and Y. S. Kivshar, Nonlinear Control of Electromagnetic Topological Edge States, Phys. Rev. Lett. 121, 163901 (2018).

[25] J. Jiang, Z. W. Guo, Y. Q. Ding, Y. Sun, Y. H. Li, H. T. Jiang, and $\mathrm{H}$. Chen, Experimental demonstration of the robust edge states in a split-ring-resonator chain, Opt. Express 26, 12891 (2018).

[26] X. Y. Xu, Q. Q. Wang, W. W. Pan, K. Sun, J. S. Xu, G. Chen, J. S. Tang, M. Gong, Y. J. Han, C. F. Li, and G. C. Guo, Measuring the Winding Number in a Large-Scale Chiral Quantum Walk, Phys. Rev. Lett. 120, 260501 (2018).

[27] M. Parto, S. Wittek, H. Hodaei, G. Harari, M. A. Bandres, J. H. Ren, M. C. Rechtsman, M. Segev, D. N. Christodoulides, and M. Khajavikhan, Edge-Mode Lasing in 1D Topological Active Array, Phys. Rev. Lett. 120, 113901 (2018).

[28] L. J. Lang, Y. Wang, H. L. Wang, and Y. D. Chong, Effects of Non-Hermiticity on Su-Schrieffer-Heeger defect states, Phys. Rev. B 98, 094307 (2018).
[29] Y. Hadad, J. C. Soric, A. B. Khanikaev, and A. Alu, Selfinduced topological protection in nonlinear circuit arrays, Nat. Electron. 1, 178 (2018).

[30] S. Xia, D. Jukić, N. Wang, D. Smirnova, L. Smirnov, L. Tang, D. Song, A. Szameit, D. Leykam, J. Xu, Z. Chen, and H. Buljan, Nontrivial coupling of light into a defect: The interplay of nonlinearity and topology, Light: Sci. Appl. 9, 147 (2020).

[31] Y. E. Kraus and O. Zilberberg, Topological Equivalence between the Fibonacci Quasicrystal and the Harper Model, Phys. Rev. Lett. 109, 116404 (2012).

[32] A. Dareau, E. Levy, M. Bosch Aguilera, R. Bouganne, E. Akkermans, F. Gerbier, and J. Beugnon, Revealing the Topology of Quasicrystals with a Diffraction Experiment, Phys. Rev. Lett. 119, 215304 (2017).

[33] Z. W. Guo, H. T. Jiang, Y. Sun, Y. H. Li, and H. Chen, Asymmetric topological edge states in a quasiperiodic Harper chain composed of split-ring resonators, Opt. Lett. 43, 5142 (2018).

[34] A. Y. Kitaev, Unpaired Majorana fermions in quantum wires, Phys. Usp. 44, 131 (2001).

[35] L. Fu and C. L. Kane, Superconducting Proximity Effect and Majorana Fermions at the Surface of a Topological Insulator, Phys. Rev. Lett. 100, 096407 (2008).

[36] W. DeGottardi, D. Sen, and S. Vishveshwara, Topological phases, Majorana modes and quench dynamics in a spin ladder system, New J. Phys. 13, 065028 (2011).

[37] V. Mourik, K. Zuo, S. M. Frolov, S. R. Plissard, E. P. A M. Bakkers, and L. P. Kouwenhoven, Signatures of Majorana fermions in hybrid superconductor-semiconductor nanowire devices, Science 336, 1003 (2012).

[38] Z. Wang, Q.-F. Liang, D.-X. Yao, and X. Hu, Viewing Majorana bound states by Rabi oscillations Sci. Rep. 5, 11686 (2015).

[39] K. Padavić, S. S. Hegde, W. DeGottardi, and S. Vishveshwara, Topological phases, edge modes, and the Hofstadter butterfly in coupled Su-Schrieffer-Heeger systems, Phys. Rev. B 98, 024205 (2018).

[40] T. Kawakami and X. Hu, Evolution of Density of States and a Spin-Resolved Checkerboard-Type Pattern Associated with the Majorana Bound State, Phys. Rev. Lett. 115, 177001 (2015).

[41] S. R. Elliott and M. Franz, Colloquium: Majorana fermions in nuclear, particle, and solid-state physics, Rev. Mod. Phys. 87, 137 (2015).

[42] M. Wimmer, A. R. Akhmerov, M. V. Medvedyeva, J. Tworzydło, and C. W. J. Beenakker, Majorana Bound States without Vortices in Topological Superconductors with Electrostatic Defects, Phys. Rev. Lett. 105, 046803 (2010).

[43] A. Kundu and B. Seradjeh, Transport Signatures of Floquet Majorana Fermions in Driven Topological Superconductors, Phys. Rev. Lett. 111, 136402 (2013).

[44] E. Prodan, K. Dobiszewski, A. Kanwal, J. Palmieri, and C. Prodan, Dynamical Majorana edge modes in a broad class of topological mechanical systems, Nat. Commun. 8, 14587 (2017).

[45] A. McDonald, T. Pereg-Barnea, and A. A. Clerk, Phasedependent chiral transport and effective non-Hermitian dynamics in a Bosonic Kitaev-Majorana chain, Phys. Rev. X 8, 041031 (2018).

[46] C. E. Bardyn and A. İmamoǧlu, Majorana-like Modes of Light in a One-Dimensional Array of Nonlinear Cavities, Phys. Rev. Lett. 109, 253606 (2012). 
[47] A. Cottet, T. Kontos, and B. Douçot, Squeezing light with Majorana fermions, Phys. Rev. B 88, 195415 (2013).

[48] B. M. Rodríguez-Lara and H. M. Moya-Cessa, Optical simulation of Majorana physics, Phys. Rev. A 89, 015803 (2014).

[49] A. Poddubny, A. Miroshnichenko, A. Slobozhanyuk, and Y. Kivshar, Topological Majorana states in zigzag chains of plasmonic nanoparticles, ACS Photon. 1, 101 (2014).

[50] I. S. Sinev, I. S. Mukhin, A. P. Slobozhanyuk, A. N. Poddubny, A. E. Miroshnichenko, A. K. S amusev, and Y. S. Kivshar, Mapping plasmonic topological states at the nanoscale, Nanoscale 7, 11904 (2015).

[51] R. Keil, C. Noh, A. Rail, S. Stützer, S. Nolte, D. G. Angelakis, and A. Szameit, Optical simulation of charge conservation violation and Majorana dynamics, Optica 2, 454 (2015).

[52] A. P. Slobozhanyuk, A. N. Poddubny, A. E. Miroshnichenko, P. A. Belov, and Y. S. Kivshar, Subwavelength Topological Edge States in Optically Resonant Dielectric Structures, Phys. Rev. Lett. 114, 123901 (2015).

[53] J. S. Xu, K. Sun, Y. J. Han, C. F. Li, J. K. Pachos, and G. C. Guo, Simulating the exchange of Majorana zero modes with a photonic system, Nat. Commun. 7, 13194 (2016).

[54] C. Li and A. E. Miroshnichenko, Extended SSH model: Nonlocal couplings and non-monotonous edge states, Physics 1, 2 (2019).

[55] Z. W. Guo, H. T. Jiang, Y. H. Li, H. Chen, and G. S. Agarwal, Enhancement of electromagnetically induced transparency in metamaterials using long range coupling mediated by a hyperbolic material, Opt. Express 26, 627 (2018).

[56] W. Tan, Y. Sun, H. Chen, and S. Q. Shen, Photonic simulation of topological excitations in metamaterials, Sci. Rep. 4, 3842 (2014).

[57] E. Tatarschuk, N. Gneiding, F. Hesmer, A. Radkovskaya, and E. Shamonina, Mapping inter-element coupling in metamaterials: Scaling down to infrared, J. Appl. Phys. 111, 094904 (2012).

[58] L. D. Landau and E. M. Lifschitz, Electrodynamics of Continuous Media (Pergamon, Oxford, 1984).

[59] K. Simonyi, Foundations of Electrical Engineering (Pergamon, Oxford, 1963).

[60] D. R. Hofstadter, Energy levels and wave functions of Bloch electrons in rational and irrational magnetic fields, Phys. Rev. B 14, 2239 (1976).

[61] X. Ni, K. Chen, M. Weiner, D. J. Apigo, C. Prodan, A. Alù, E. Prodan, and A. B. Khanikaev, Observation of Hofstadter butterfly and topological edge states in reconfigurable quasi-periodic acoustic crystals, Commun. Phys. 2, 55 (2019).

[62] M. K. Liu, D. A. Powell, Y. Zarate, and I. V. Shadrivov, Huygens' Metadevices for Parametric Waves, Phys. Rev. X 8, 031077 (2018).

[63] J. Ran, Y. W. Zhang, X. D. Chen, and H. Chen, Frequency mixer based on doppler effect, IEEE Microw. Wireless Compon. Lett. 28, 43 (2017).

[64] Z. W. Guo, H. T. Jiang, Y. Sun, Y. H. Li, and H. Chen, Actively controlling the topological transition of dispersion based on electrically controllable metamaterials, Appl. Sci. 8, 596 (2018).

[65] C. W. Peterson, W. A. Benalcazar, T. L. Hughes, and G. Bahl, A quantized microwave quadrupole insulator with topologically protected corner states, Nature (London) 555, 346 (2018).
[66] X. D. Chen, W. M. Deng, F. L. Shi, F. L. Zhao, M. Chen, and J. W. Dong, Direct Observation of Corner States in SecondOrder Topological Photonic Crystal Slabs, Phys. Rev. Lett. 122, 233902 (2019).

[67] B. Y. Xie, G. X. Su, H. F. Wang, H. Su, X. P. Shen, P. Zhan, M. H. Lu, Z. L. Wang, and Y. F. Chen, Visualization of Higher-Order Topological Insulating Phases in Two-Dimensional Dielectric Photonic Crystals, Phys. Rev. Lett. 122, 233903 (2019).

[68] J. Wiersig, Enhancing the Sensitivity of Frequency and Energy Splitting Detection by Using Exceptional Points: Application to Microcavity Sensors for Single-Particle Detection, Phys. Rev. Lett. 112, 203901 (2014).

[69] S. Assawaworrarit, X. F Yu, and S. H. Fan, Robust wireless power transfer using a nonlinear parity-time-symmetric circuit, Nature (London) 546, 387 (2017).

[70] M. A. Miri and A. Alu, Exceptional points in optics and photonics, Science 363, eaar7709 (2019).

[71] Ş. K. Özdemir, S. Rotter, F. Nori, and L. Yang, Parity-time symmetry and exceptional points in photonics, Nat. Mater. 18, 783 (2019).

[72] H. Hodaei, A. U. Hassan, S. Wittek, H. Garcia-Gracia, R. Elganainy, D. N. Christodoulides, and M. Khajavikhan, Enhanced sensitivity at higher-order exceptional points, Nature (London) 548, 187 (2017).

[73] W. J. Chen, Ş. K. Özdemir, G. M. Zhao, J. Wiersig, and L. Yang, Exceptional points enhance sensing in an optical microcavity, Nature (London) 548, 192 (2017).

[74] Q. Zhong, J. Ren, M. Khajavikhan, D. N. Christodoulides, Ş. K. Özdemir, and R. El-Ganainy, Sensing with Exceptional Surfaces in Order to Combine Sensitivity with Robustness, Phys. Rev. Lett. 122, 153902 (2019).

[75] J. C. Budich and E. J. Bergholtz, Non-Hermitian Topological Sensors, Phys. Rev. Lett. 125, 180403 (2020).

[76] M. D. Wang, L. P. Ye, J. Christensen, and Z. Y. Liu, Valley Physics in Non-Hermitian Artificial Acoustic Boron Nitride, Phys. Rev. Lett. 120, 246601 (2018).

[77] W. W. Zhu, X. S. Fang, D. T. Li, Y. Sun, Y. Li, Y. Jing, and H. Chen, Simultaneous Observation of a Topological Edge State and Exceptional Point in an Open and NonHermitian Acoustic System, Phys. Rev. Lett. 121, 124501 (2018).

[78] W. Song, W. Z. Sun, C. Chen, Q. H. Song, S. M. Xiao, S. N. Zhu, and T. Li, Breakup and Recovery of Topological Zero Modes in Finite Non-Hermitian Optical Lattices, Phys. Rev. Lett. 123, 165701 (2019).

[79] X.-C. Sun and X. Hu, Topological ring-cavity laser formed by honeycomb photonic crystals, arXiv:1906.02464.

[80] J. Song, F. Q. Yang, Z. W. Guo, X. Wu, K. J. Zhu, J. Jiang, Y. Sun, Y. H. Li, H. T. Jiang, and H. Chen, Wireless Power Transfer via Topological Modes in Dimer Chains, Phys. Rev. Appl. 15, 014009 (2021).

[81] P. Y. Chen, M. Sakhdari, M. Hajizadegan, Q. S. Cui, M. M.-C. Cheng, R. El-Ganainy, and A. Alù, Generalized paritytime symmetry condition for enhanced sensor telemetry, Nat. Electron. 1, 297 (2018).

[82] Z. Dong, Z. P. Li, F. Yang, C. W. Qiu, and J. S. Ho, Sensitive readout of implantable microsensors using a wireless system locked to an exceptional point, Nat. Electron. 2, 335 (2019). 